\title{
Effect of Integrated Nitrogen Management on Macronutrient Availability under Cauliflower (Brassica oleracea var. botrytis L.)
}

\author{
Harmandeep Singh Chahal, Satnam Singh, Iqbal Singh Dhillon and Simerpreet Kaur*
}

PG Department of Agriculture, Khalsa College, Amritsar-143005, Punjab, India

*Corresponding author

\section{Keywords}

Nitrogen management, Inorganic fertilizers, Vermicompost, FYM, Cauliflower

Article Info

Accepted:

12 March 2019

Available Online:

10 April 2019

\section{A B S T R A C T}

This study investigates the effect of integrated nitrogen management (INM) on yield and soil properties under cauliflower (Brassica oleracea var. botrytis L.) cv. Nuzi Snow White at Students' Research Farm, P.G. Department of Agriculture, Khalsa College, Amritsar during Rabi season of 2017-18. For INM studies, organic manures viz., vermicompost (VC), farmyard manure (FYM) and recommended dose of nitrogenous fertilizers (RDNF) was employed. The experiment was laid out in Randomized Block Design (RBD) with eight treatments comprised of $\mathrm{T}_{1}$ : Control, $\mathrm{T}_{2}: 100 \% \mathrm{RDF}, \mathrm{T}_{3}: 75 \% \mathrm{RDNF}+25 \% \mathrm{~N}$ (VC), $\mathrm{T}_{4}: 50 \% \mathrm{RDNF}+50 \% \mathrm{~N}(\mathrm{VC}), \mathrm{T}_{5}: 75 \% \mathrm{RDNF}+25 \% \mathrm{~N}(\mathrm{FYM}), \mathrm{T}_{6}: 50 \% \mathrm{RDNF}+$ $50 \% \mathrm{~N}$ (FYM), $\mathrm{T}_{7}: 50 \% \mathrm{RDNF}+25 \% \mathrm{~N}(\mathrm{VC})+25 \% \mathrm{~N}(\mathrm{FYM}), \mathrm{T}_{8}: 75 \% \mathrm{RDNF}+12.5 \%$ $\mathrm{N}(\mathrm{VC})+12.5 \% \mathrm{~N}(\mathrm{FYM})$. The availability of macro and micronutrients were significantly improved by integrated use of nutrient sources. Maximum values of available $\mathrm{N}, \mathrm{P}, \mathrm{K}$ and $\mathrm{S}$ were observed to be $255.90 \mathrm{~kg} \mathrm{ha}^{-1}, 25.18 \mathrm{~kg} \mathrm{ha}^{-1}, 308.65 \mathrm{~kg} \mathrm{ha}^{-1}$ and 11.97 $\mathrm{kg} \mathrm{ha}^{-1}$, respectively in a treatment where $50 \%$ recommended nitrogen was supplied through VC $\left(\mathrm{T}_{4}\right)$. Similarly, available micronutrients $(\mathrm{Zn}, \mathrm{Fe}, \mathrm{Cu}$ and $\mathrm{Mn})$ were also found highest in treatment $\mathrm{T}_{4}$. Exchangeable $\mathrm{Ca}$ and $\mathrm{Mg}$ in soil varied from 58.47 to $33.47 \mathrm{mg} \mathrm{kg}$ ${ }^{1}$ and 6.73 to $11.87 \mathrm{mg} \mathrm{kg}^{-1}$, respectively. Values regarding plant growth parameters, yield as well as nutrient uptake were noticed highest in $\mathrm{T}_{2}(100 \%$ NPK through inorganic fertilizers) which was at par with $\mathrm{T}_{3}$ in which $25 \% \mathrm{~N}$ was substituted through VC. Integrated application of organic inputs along with $25 \%$ reduced chemical fertilizers proved to enhance the soil health while sustaining crop productivity.

\section{Introduction}

Cauliflower (Brassica oleracea var. botrytis) is one of the most important vegetable crops belonging to the family Brassicaceae. It is widely cultivated all over world for its nutritive values, high productivity and wider adaptability under different ecological conditions. Cauliflower contains various kinds of vitamins, especially vitamin C. It also contains minerals like potassium, sodium, calcium, iron, phosphorus and magnesium (Ogbede et al., 2015). Cauliflower is a heavy feeder of mineral elements, it removes a large amount of macronutrients from the soil, especially nitrogen. The use of nitrogenous fertilizers upsurges tremendously in order to fulfil growing food needs since the green revolution. Applicability of nitrogen- 
containing fertilizer at or below than optimum levels help in proper build up of organic nitrogen in the soil whereas excessive and imbalance use of synthetic nitrogen sources cause loss of native nitrogen that ultimately makes soil devoid of good structure as well as soil biomass (Singh, 2018). Nitrogen applied through fertilizers of ammonical nature produce acidic conditions in the soil (Fageria et al., 2010). The annual consumption of fertilizers increased from about 255.36 LMT in 2012-13 to 259.49 LMT in 2016-17 (Anonymous, 2017). In general agriculture, the use of chemical fertilizers cannot be ruled out completely. However, there is a need for the integrated application of different sources of nutrients for sustaining the required crop productivity by integrated nutrient management. The concept of integrated nutrient management requires the optimum use of organic, inorganic and bio-sources of plant nutrients (Tekasangla et al., 2015). Organic manures like FYM, poultry manure, vermicompost have traditionally used by farmers in different regions of Punjab. In the past, the integrated use of organic materials and inorganic nitrogenous fertilizers have received notable attention to meet the farmer's economic need as well as maintaining ecological conditions on the long-term basis (Kumar et al., 2007). Keeping the above in view, the present work was undertaken to study the impact of different combination of nutrient sources on macronutrient availability under cauliflower.

\section{Materials and Methods}

The field experiment was conducted during Rabi season (Aug to Dec) of 2017 at Students' Research Farm, Khalsa College, Amritsar, India on sandy loam, moderately alkaline ( $\mathrm{pH} 8.22)$, medium in organic carbon $(0.45 \%)$, available $\mathrm{P}\left(20.53 \mathrm{~kg} \mathrm{ha}^{-1}\right)$ and available $\mathrm{K}\left(261.36 \mathrm{~kg} \mathrm{ha}^{-1}\right)$ and low in available $\mathrm{N}$ (198.21 $\mathrm{kg} \mathrm{ha}^{-1}$ ) soil. Organic manures viz., farmyard manure (FYM), vermicompost (VC) containing various macro and micronutrients (Table 1) and recommended dose of nitrogenous fertilizers (RDNF) were applied according to the treatments. Organic wastes used for vermicomposting includes waste vegetables, cow dung, green weeds etc. having high nitrogen content. FYM was prepared from cow dung and other farm waste.

The experiment was laid out in Randomized Block Design (RBD) with the three replications. A total of eight treatments; $\mathrm{T}_{1}$ Control, $\mathrm{T}_{2}-100 \%$ recommended NPK through fertilizers, $\mathrm{T}_{3}-75 \% \mathrm{RDNF}+25 \% \mathrm{~N}$ through $\mathrm{VC}, \mathrm{T}_{4}-50 \% \mathrm{RDNF}+50 \% \mathrm{~N}$ through $\mathrm{VC}, \mathrm{T}_{5}-75 \% \mathrm{RDNF}+25 \% \mathrm{~N}$ through FYM, $\mathrm{T}_{6}-50 \% \mathrm{RDNF}+50 \% \mathrm{~N}$ through $\mathrm{FYM}_{1} \mathrm{~T}_{7}-50 \% \mathrm{RDNF}+25 \% \mathrm{~N}$ through $\mathrm{VC}+25 \% \mathrm{~N}$ through $\mathrm{FYM}, \mathrm{T}_{8}-75 \%$ $\mathrm{RDNF}+12.5 \% \mathrm{~N}$ through $\mathrm{VC}+12.5 \% \mathrm{~N}$ through FYM were evaluated for nutrient management. Organic manures were incorporated in soil 15 days before transplanting. Cauliflower seedlings of the cultivar Nuzi Snow White were procured from KVK, Amritsar. Seedlings were transplanted in $2.7 \mathrm{~m} \times 2.7 \mathrm{~m}$ plots with both ways spacing of $45 \mathrm{~cm}$. Fertilizers used were urea $(46 \% \mathrm{~N})$, single superphosphate $(16 \% \mathrm{P})$ and muriate of potash $(60 \% \mathrm{~K})$. All the dose of $\mathrm{P}, \mathrm{K}$ and half dose of $\mathrm{N}$ was applied as basal dressing and the remainder of $\mathrm{N}$ was top-dressed after 4 weeks of transplanting as recommended in the package of practices for cultivation of vegetables published by Punjab Agricultural University, Ludhiana.

Soil samples were taken from all the replications before starting the experiment and after the harvest of cauliflower from each treatment. Soil Samples were air dried and ground to pass through a $2 \mathrm{~mm}$ sieve. Soil samples were analysed for $\mathrm{pH}$ and $\mathrm{EC}$ in 1:2 soil: water suspension, organic carbon 
(Walkley and Black, 1934), cation exchange capacity (Jackson, 1987), available N (Subbiah and Asija, 1965), available P (Olsen, 1954), available $\mathrm{K}$, exchangeable $\mathrm{Ca}$ and exchangeable Mg (Merwin and Peech, 1951), available $\mathrm{S}$ (Chesnin and Yien, 1950) and DTPA-extractable $\mathrm{Zn}, \mathrm{Fe}, \mathrm{Cu}$ and $\mathrm{Mn}$ by the method of Lindsay and Norvell (1978). Total N, P (Chapman and Pratt, 1961; Jackson, 1987) and K (Jackson, 1987) uptake in plant samples was analyzed after the harvest. Agronomic nitrogen use efficiency (Dilz, 1988) and apparent nitrogen recovery were calculated as described by Novoa and Loomis (1981).

\section{Results and Discussion}

Use of organic manures and inorganic fertilizers showed significant impact on yield and other attributes of cauliflower. Highest plant height (at 45 DAT - $32.81 \mathrm{~cm}$ and at harvest - $76.82 \mathrm{~cm}$ ), plant spread (at $45 \mathrm{DAT}$ $159.37 \mathrm{~cm}$ and at harvest $-215.24 \mathrm{~cm})$, no. of leaves plant ${ }^{-1}(19.45)$ and leaf area (1132.00 $\mathrm{cm}^{2}$ ) was recorded under application of $100 \%$ NPK through inorganic fertilizers which was statistically at par with treatment $\mathrm{T}_{3}$ in which $25 \% \mathrm{~N}$ was applied through VC (Table 1).

This could be attributed to higher dose of inorganic nitrogen which is associated with increase in protoplasm, cell division and cell enlargement resulting in taller plants (Kumari, 2017). There was increase in number of leaves with the increase in nitrogen doses because nitrogen promotes the apical branching and hence more number of leaves appeared on plant (Kebrom, 2017).

Higher leaf area was also related to addition of nitrogen in different doses and nitrogen mediates cell expansion (Chaudhary et al., 2015). Significant increase in plant growth in vermicompost treated plots over FYM treated plots may have been due to its faster mineralization of nutrients from vermicompost (Joshi et al., 2015) (Table 2).

The observation on stalk length, girth of stem, dry matter accumulation and yield showed an increasing trend with higher level inorganic nitrogen doses (Table 3). The highest stalk length $(14.63 \mathrm{~cm})$ and girth of stem $(12.47$ $\mathrm{cm})$ was recorded with application of $100 \%$ RDF alone which was at par with treatment $\mathrm{T}_{3}$. Stalk length as well as girth of stem was increased due to less retention in the roots and more translocation of nutrients to aerial parts for synthesis of protoplasmic protein and other metabolites (Rather et al., 2018).

Dry matter accumulation varied from $18.23 \mathrm{q}$ ha $^{-1}$ to $34.49 \mathrm{q} \mathrm{ha}^{-1}$. Treatments $\mathrm{T}_{7}$ and $\mathrm{T}_{6}$ in which $50 \% \mathrm{~N}$ was applied through $\mathrm{VC}$ and FYM were at par and inferior to all treatments except the control. The weight gain of plant or plant organs indicated that the plant growth and development occurred by increasing the size and volume of the cell due to release of nutrients (Kumari, 2017). Yield of cauliflower ranged from $378.86 \mathrm{q} \mathrm{ha}^{-1}$ to $202.58 \mathrm{q} \mathrm{ha}^{-1}$ in all the treatments. The effect of combined use of organic manures have not resulted significant influence on yield of cauliflower and highest yield was found under 100\% RDF treated plots, however it was statistically at par with $T_{3}$ treatment. Nitrogen being the major constituent of chlorophyll, amino acids and proteins as well as phosphorus being the component of energy compounds viz., ATP, NADP and potassium regulates the activity of various enzymes involved in photosynthesis and $\mathrm{CO}_{2}$ fixation (Ohyama, 2010). It could have promoted satisfactory plant growth, yield structure and finally to cauliflower yield under adequate and balanced supply of nutrients at maximum level.

Application of $100 \%$ RDF resulted in highest $\mathrm{N}\left(82.12 \mathrm{~kg} \mathrm{ha}^{-1}\right), \mathrm{P}\left(12.94 \mathrm{~kg} \mathrm{ha}^{-1}\right)$ and $\mathrm{K}$ (60.21 $\mathrm{kg} \mathrm{ha}^{-1}$ ) uptake by cauliflower which 
was found to be statistically similar to treatment $\mathrm{T}_{3}$ in which $25 \% \mathrm{~N}$ was substituted through VC (Table 4). Least N, P and K uptake was found in control due to less growth of plants in such plots. The increase in uptake of nitrogen with application of inorganic fertilizers as well as in combination of organic manures was consistent with the findings of Bozkurt et al., (2011) who concluded that this nitrogen was further utilized for metabolism of various substances required for growth of plants which produced more dry matter. The ability of a plant to take up phosphorus is largely due to its root distribution relative to phosphorus as it is relatively immobile in the soil. Application of organic manures might have improved the soil environment, which encouraged proliferous root system resulting in better absorption of water and nutrients from lower layers and thus resulting in higher yield and nutrient uptake (Devi et al., 2017). Higher potassium uptake in all plots over the control might be due to the enhanced number of small root hairs resulting from more growth of plant which in turn increased the absorbing ability (Reza et al., 2016).

Likewise, Maximum agronomic nitrogen use efficiency was recorded under $100 \%$ RDF (141.02 $\left.\mathrm{kg} \mathrm{ha}^{-1}\right)$ and minimum value of agronomic nitrogen use efficiency in treatments $\mathrm{T}_{4}, \mathrm{~T}_{7}$ and $\mathrm{T}_{6}$ in which $50 \% \mathrm{~N}$ was applied through VC and FYM was due to lower yield in such treatments (Table 5). Similar trend was observed for apparent nitrogen recovery (\%). Kumar and Mukhopadhyay (2017) also reported an increase of agronomic nitrogen use efficiency and apparent nitrogen recovery with yield of cauliflower with same dose of nitrogen applied.

Apart from all, there was no significant difference in soil $\mathrm{pH}$ and soil EC among different treatments was observed (Table 6).
Maximum organic carbon $(0.56 \%)$ and CEC [11.51 $\left.\mathrm{cmol}\left(\mathrm{p}^{+}\right) \mathrm{kg}^{-1}\right]$ was recorded in treatment $\mathrm{T}_{6}[50 \% \mathrm{RDNF}+\% 50 \% \mathrm{~N}$ $(\mathrm{FYM})]$ that was at par with treatments $\mathrm{T}_{7}$ $[50 \%$ RDNF $+25 \%(\mathrm{VC})+25 \%(\mathrm{FYM})]$ and $\mathrm{T}_{4}[50 \% \mathrm{RDNF}+50 \%(\mathrm{VC})]$. The increase in soil organic carbon content with the application of FYM and VC may be attributed due to direct incorporation of these organic materials in the soil and the subsequent decomposition of these materials result in enhanced organic carbon content of the soil. Similar trend was also reported by Merentola et al., (2012). Application of organic inputs increased the organic carbon stock in soil which, ultimately resulted in higher cation exchange capacity (CEC). The findings are well supported by those reported by Scotti et al., (2015).

Available $\mathrm{N}, \mathrm{P}, \mathrm{K}$ and $\mathrm{S}$ mean values were lower in control plots $\left(\mathrm{T}_{1}\right)$ and it increased significantly with the application of chemical fertilizers and organic manures (Table 7). Maximum available $\mathrm{N}, \mathrm{P}, \mathrm{K}$ and $\mathrm{S}$ was observed in treatment $\mathrm{T}_{4}$ which was at par with treatments $T_{7}$ and $T_{6}$. Higher availability of nitrogen was observed in case of treatments that received combined application of organic manures and inorganic fertilizers might be partly due to release of native soil nitrogen and partly due to mineralization of nutrients from organic manures (Singh, 2018). Available P varied from $13.42 \mathrm{~kg} \mathrm{ha}^{-1}$ under control to $25.18 \mathrm{~kg} \mathrm{ha}^{-1}$. The conjunctive use of inorganic and organic sources increased the phosphorus availability by reducing the fixation of water soluble $\mathrm{P}$ and by increasing mineralization that resulted in more availability of phosphorus (Singh et al., 2015). Available potassium was improved in INM plots over the control. This might be due to the organic acids released during decomposition of manures mobilize the native or non-exchangeable forms of potassium, so that it will be readily available Chander et al., (2010). 
Table.1 Nutrient composition of vermicompost and farmyard manure

\begin{tabular}{|l|c|c|c|c|c|c|c|c|c|c|}
\hline \multirow{2}{*}{ Source } & \multicolumn{4}{|c|}{ Macronutrients } & \multicolumn{4}{c|}{ Micronutrients } \\
\cline { 2 - 11 } & \multicolumn{4}{|c|}{ Per cent } & \multicolumn{4}{c|}{ mg kg $^{-1}$} & \multicolumn{4}{c|}{} \\
\cline { 2 - 11 } & $\mathrm{N}$ & $\mathrm{P}$ & $\mathrm{K}$ & $\mathrm{S}$ & $\mathrm{Ca}$ & $\mathrm{Mg}$ & $\mathrm{Zn}$ & $\mathrm{Fe}$ & $\mathrm{Cu}$ & $\mathrm{Mn}$ \\
\hline VC & 1.68 & 1.05 & 1.21 & 0.57 & 64.30 & 19.97 & 22.14 & 76.32 & 4.08 & 121.16 \\
\hline FYM & 0.87 & 0.49 & 0.77 & 0.42 & 47.43 & 16.81 & 15.43 & 67.83 & 2.97 & 103.84 \\
\hline
\end{tabular}

Table.2 Effect of integrated nitrogen management on plant height, plant spread, no of leaves plant $^{-1}$ and leaf area

\begin{tabular}{|c|c|c|c|c|c|c|c|}
\hline \multirow[t]{2}{*}{ Symbol } & \multirow[t]{2}{*}{ Treatments } & \multicolumn{2}{|c|}{ Plant height (cm) } & \multicolumn{2}{|c|}{ Plant spread (cm) } & \multirow{2}{*}{$\begin{array}{l}\text { No. of } \\
\text { leaves } \\
\text { plant }^{-1}\end{array}$} & \multirow{2}{*}{$\begin{array}{l}\text { Leaf } \\
\text { Area } \\
\left(\mathrm{cm}^{2}\right)\end{array}$} \\
\hline & & 45 DAT & At Harvest & $\begin{array}{c}45 \\
\text { DAT }\end{array}$ & At Harvest & & \\
\hline$T_{1}$ & Control & 20.08 & 41.62 & 100.67 & 123.28 & 9.35 & 594.67 \\
\hline $\mathbf{T}_{2}$ & $100 \%$ RDF & 32.81 & 76.82 & 159.37 & 215.24 & 19.45 & 1132.00 \\
\hline $\mathbf{T}_{3}$ & $\begin{array}{l}75 \% \mathrm{RDNF}+25 \% \mathrm{~N} \\
(\mathrm{VC})\end{array}$ & 30.47 & 73.91 & 148.10 & 200.26 & 18.27 & 1081.00 \\
\hline $\mathbf{T}_{4}$ & $\begin{array}{l}50 \% \mathrm{RDNF}+50 \% \mathrm{~N} \\
(\mathrm{VC})\end{array}$ & 27.31 & 64.59 & 132.22 & 175.33 & 16.23 & 978.00 \\
\hline $\mathbf{T}_{5}$ & $\begin{array}{l}75 \% \mathrm{RDNF}+25 \% \mathrm{~N} \\
(\mathrm{FYM})\end{array}$ & 29.17 & 69.49 & 142.96 & 189.47 & 17.31 & 1028.33 \\
\hline $\mathbf{T}_{6}$ & $\begin{array}{l}50 \% \text { RDNF + 50\% N } \\
\text { (FYM) }\end{array}$ & 25.27 & 58.79 & 127.90 & 166.27 & 15.15 & 917.00 \\
\hline $\mathbf{T}_{7}$ & $\begin{array}{l}50 \% \mathrm{RDNF}+25 \% \mathrm{~N} \\
(\mathrm{VC})+25 \% \mathrm{~N}(\mathrm{FYM})\end{array}$ & 26.30 & 61.40 & 130.38 & 171.80 & 15.77 & 948.00 \\
\hline $\mathbf{T}_{8}$ & $\begin{array}{l}75 \% \mathrm{RDNF}+12.5 \% \mathrm{~N} \\
(\mathrm{VC})+12.5 \% \mathrm{~N}(\mathrm{FYM})\end{array}$ & 29.86 & 70.60 & 146.29 & 195.55 & 17.72 & 1057.00 \\
\hline & $\mathrm{CD}(\mathrm{p}=0.05)$ & 2.51 & 5.94 & 12.15 & 16.43 & 1.42 & 64.9 \\
\hline
\end{tabular}

Table.3 Effect of integrated nitrogen management on stalk length, girth of stem, dry matter and yield

\begin{tabular}{|c|c|c|c|c|c|}
\hline $\begin{array}{l}\text { Symbo } \\
\quad 1\end{array}$ & Treatments & $\begin{array}{c}\text { Stalk } \\
\text { Length } \\
\text { (cm) }\end{array}$ & $\begin{array}{l}\text { Girth of } \\
\text { stem }(\mathbf{c m})\end{array}$ & $\begin{array}{l}\text { Dry Matter } \\
\quad\left(\mathbf{q} \mathbf{h a}^{-1}\right)\end{array}$ & $\begin{array}{l}\text { Yield } \\
\left(\mathbf{q} \text { ha }^{-1}\right)\end{array}$ \\
\hline $\mathbf{T}_{1}$ & Control & 8.17 & 7.17 & 18.23 & 202.58 \\
\hline $\mathbf{T}_{2}$ & $100 \% \mathrm{RDF}$ & 14.63 & 12.47 & 34.49 & 378.86 \\
\hline $\mathbf{T}_{3}$ & $75 \%$ RDNF + $25 \% \mathrm{~N}(\mathrm{VC})$ & 13.61 & 11.76 & 32.79 & 357.28 \\
\hline $\mathbf{T}_{4}$ & $50 \%$ RDNF + 50\% N (VC) & 12.05 & 10.42 & 28.90 & 319.18 \\
\hline $\mathbf{T}_{5}$ & $75 \%$ RDNF + 25\% N (FYM) & 12.94 & 11.28 & 30.57 & 346.74 \\
\hline $\mathbf{T}_{6}$ & $50 \%$ RDNF + 50\% N (FYM) & 11.43 & 10.04 & 26.65 & 311.78 \\
\hline $\mathbf{T}_{7}$ & $\begin{array}{l}50 \% \mathrm{RDNF}+25 \% \mathrm{~N}(\mathrm{VC})+ \\
25 \% \mathrm{~N}(\mathrm{FYM})\end{array}$ & 11.67 & 10.29 & 28.04 & 315.07 \\
\hline \multirow[t]{2}{*}{$\mathbf{T}_{8}$} & $\begin{array}{l}75 \% \text { RDNF }+12.5 \% \mathrm{~N}(\mathrm{VC})+ \\
12.5 \% \mathrm{~N}(\mathrm{FYM})\end{array}$ & 13.22 & 11.42 & 31.94 & 349.24 \\
\hline & $\mathrm{CD}(\mathrm{p}=0.05)$ & 1.10 & 0.95 & 2.52 & 29.45 \\
\hline
\end{tabular}


Table.4 Effect of integrated nitrogen management on N, P and K uptake by cauliflower

\begin{tabular}{|c|c|c|c|c|}
\hline Symbol & Treatments & $\begin{array}{c}\text { N uptake (kg } \\
\left.\text { ha }^{-1}\right)\end{array}$ & $\begin{array}{c}\text { P uptake (kg } \\
\left.\text { ha }^{-1}\right)\end{array}$ & $\begin{array}{c}\text { K uptake (kg } \\
\left.\text { ha }^{-1}\right)\end{array}$ \\
\hline $\mathbf{T}_{1}$ & Control & 36.15 & 6.37 & 27.61 \\
\hline $\mathbf{T}_{2}$ & $100 \% \mathrm{RDF}$ & 82.12 & 12.94 & 60.21 \\
\hline $\mathbf{T}_{3}$ & $75 \% \mathrm{RDNF}+25 \% \mathrm{~N}(\mathrm{VC})$ & 76.64 & 12.06 & 57.50 \\
\hline $\mathbf{T}_{4}$ & $50 \%$ RDNF + 50\% N (VC) & 66.63 & 10.24 & 51.49 \\
\hline $\mathbf{T}_{5}$ & $75 \%$ RDNF + 25\% N (FYM $)$ & 70.74 & 11.13 & 54.40 \\
\hline $\mathbf{T}_{6}$ & $50 \%$ RDNF + 50\% N (FYM) & 60.85 & 9.36 & 48.25 \\
\hline $\mathbf{T}_{7}$ & $50 \% \mathrm{RDNF}+25 \% \mathrm{~N}(\mathrm{VC})+25 \% \mathrm{~N}(\mathrm{FYM})$ & 64.84 & 9.75 & 49.42 \\
\hline \multirow[t]{2}{*}{$\mathbf{T}_{8}$} & $75 \% \mathrm{RDNF}+12.5 \% \mathrm{~N}(\mathrm{VC})+12.5 \% \mathrm{~N}(\mathrm{FYM})$ & 73.41 & 11.62 & 56.12 \\
\hline & $\mathrm{CD}(\mathrm{p}=0.05)$ & 6.06 & 0.96 & 3.76 \\
\hline
\end{tabular}

Table.5 Effect of integrated nitrogen management on agronomic nitrogen use efficiency and apparent nitrogen recovery

\begin{tabular}{|c|c|c|c|}
\hline Symbol & Treatments & $\begin{array}{l}\text { Agronomic nitrogen use } \\
\text { efficiency (kg yield } \mathrm{kg}^{-1} \mathrm{~N} \\
\text { applied) }\end{array}$ & $\begin{array}{c}\text { Apparent nitrogen recovery } \\
(\%)\end{array}$ \\
\hline $\mathbf{T}_{1}$ & Control & --- & --- \\
\hline $\mathbf{T}_{2}$ & $100 \% \mathrm{RDF}$ & 141.02 & 36.78 \\
\hline $\mathbf{T}_{3}$ & $75 \% \mathrm{RDNF}+25 \% \mathrm{~N}(\mathrm{VC})$ & 123.77 & 32.39 \\
\hline $\mathbf{T}_{4}$ & $50 \%$ RDNF + 50\% N (VC) & 93.28 & 24.38 \\
\hline $\mathbf{T}_{5}$ & $75 \%$ RDNF + 25\% N (FYM) & 115.33 & 27.67 \\
\hline $\mathbf{T}_{6}$ & $50 \% \mathrm{RDNF}+50 \% \mathrm{~N}(\mathrm{FYM})$ & 87.36 & 19.76 \\
\hline $\mathbf{T}_{7}$ & $\begin{array}{l}50 \% \text { RDNF + 25\% N (VC) + 25\% } \\
N(\text { FYM })\end{array}$ & 89.99 & 22.95 \\
\hline \multirow[t]{2}{*}{$\mathbf{T}_{8}$} & $\begin{array}{l}75 \% \text { RDNF + } 12.5 \% \mathrm{~N}(\mathrm{VC})+ \\
12.5 \% \mathrm{~N}(\mathrm{FYM})\end{array}$ & 117.33 & 29.81 \\
\hline & $\mathrm{CD}(\mathrm{p}=0.05)$ & --- & --- \\
\hline
\end{tabular}

Table.6 Effect of integrated nitrogen management on soil pH, EC, organic carbon and cation exchange capacity

\begin{tabular}{|c|c|c|c|c|c|}
\hline Symbol & Treatments & pH & $\begin{array}{c}\mathrm{EC}\left(\mathrm{dS} \mathrm{m}^{-}\right. \\
\mathbf{1})\end{array}$ & $\begin{array}{c}\text { Organic } \\
\text { carbon } \\
(\%)\end{array}$ & $\begin{array}{c}\text { CEC cmol } \\
\left(\mathrm{p}^{+}\right) \mathrm{kg}^{-1}\end{array}$ \\
\hline $\mathbf{T}_{1}$ & Control & 8.21 & 0.31 & 0.44 & 10.20 \\
\hline $\mathbf{T}_{2}$ & $100 \% \mathrm{RDF}$ & 8.19 & 0.31 & 0.45 & 10.47 \\
\hline $\mathbf{T}_{3}$ & $75 \% \mathrm{RDNF}+25 \% \mathrm{~N}(\mathrm{VC})$ & 8.18 & 0.32 & 0.49 & 10.73 \\
\hline $\mathbf{T}_{4}$ & $50 \%$ RDNF + 50\% N (VC) & 8.16 & 0.33 & 0.53 & 11.25 \\
\hline $\mathbf{T}_{5}$ & $75 \%$ RDNF + 25\% N (FYM) & 8.16 & 0.32 & 0.51 & 10.98 \\
\hline $\mathbf{T}_{6}$ & $50 \%$ RDNF + 50\% N (FYM $)$ & 8.14 & 0.33 & 0.56 & 11.51 \\
\hline $\mathbf{T}_{7}$ & $50 \% \mathrm{RDNF}+25 \% \mathrm{~N}(\mathrm{VC})+25 \% \mathrm{~N}(\mathrm{FYM})$ & 8.15 & 0.33 & 0.55 & 11.38 \\
\hline \multirow[t]{2}{*}{$\mathbf{T}_{8}$} & $75 \% \mathrm{RDNF}+12.5 \% \mathrm{~N}(\mathrm{VC})+12.5 \% \mathrm{~N}(\mathrm{FYM})$ & 8.18 & 0.32 & 0.50 & 10.86 \\
\hline & $\mathrm{CD}(\mathrm{p}=0.05)$ & N.S & N.S & 0.03 & 0.28 \\
\hline
\end{tabular}


Table.7 Effect of integrated nitrogen management on available and exchangeable macronutrients

\begin{tabular}{|c|c|c|c|c|c|c|c|}
\hline \multirow[t]{2}{*}{ Symbol } & \multirow[t]{2}{*}{ Treatments } & \multicolumn{4}{|c|}{ Available macronutrient $\left(\mathrm{kg} \mathrm{ha}^{-1}\right)$} & \multicolumn{2}{|c|}{$\begin{array}{l}\text { Exchangeable } \\
\text { Macronutrients } \\
\left(\mathrm{mg} \mathrm{kg}^{-1}\right)\end{array}$} \\
\hline & & $\mathbf{N}$ & $\mathbf{P}$ & $\mathbf{K}$ & $\mathbf{S}$ & $\mathbf{C a}$ & Mg \\
\hline $\mathbf{T}_{1}$ & Control & 178.30 & 13.42 & 246.58 & 7.43 & 33.47 & 6.73 \\
\hline $\mathbf{T}_{2}$ & $100 \% \mathrm{RDF}$ & 222.76 & 19.94 & 278.75 & 9.07 & 46.60 & 7.37 \\
\hline $\mathbf{T}_{3}$ & $\begin{array}{l}75 \% \mathrm{RDNF}+25 \% \mathrm{~N} \\
\text { (VC) }\end{array}$ & 238.28 & 22.77 & 293.48 & 10.33 & 52.70 & 10.53 \\
\hline $\mathbf{T}_{4}$ & $\begin{array}{l}50 \% \mathrm{RDNF}+50 \% \mathrm{~N} \\
(\mathrm{VC})\end{array}$ & 255.90 & 25.18 & 308.65 & 11.97 & 58.47 & 11.87 \\
\hline $\mathbf{T}_{\mathbf{5}}$ & $\begin{array}{l}75 \% \mathrm{RDNF}+25 \% \mathrm{~N} \\
(\mathrm{FYM})\end{array}$ & 230.11 & 21.43 & 285.95 & 9.83 & 49.53 & 10.03 \\
\hline $\mathbf{T}_{6}$ & $\begin{array}{l}50 \% \mathrm{RDNF}+50 \% \mathrm{~N} \\
(\mathrm{FYM})\end{array}$ & 245.76 & 23.76 & 299.94 & 11.17 & 55.80 & 11.23 \\
\hline $\mathbf{T}_{7}$ & $\begin{array}{l}50 \% \mathrm{RDNF}+25 \% \mathrm{~N} \\
(\mathrm{VC})+25 \% \mathrm{~N}(\mathrm{FYM})\end{array}$ & 249.19 & 24.39 & 304.20 & 11.43 & 56.73 & 11.60 \\
\hline $\mathbf{T}_{8}$ & $\begin{array}{l}75 \% \mathrm{RDNF}+12.5 \% \mathrm{~N} \\
(\mathrm{VC})+12.5 \% \mathrm{~N} \\
(\mathrm{FYM})\end{array}$ & 234.99 & 22.21 & 289.23 & 10.03 & 51.53 & 10.33 \\
\hline & $\mathrm{CD}(\mathrm{p}=0.05)$ & 10.44 & 1.50 & 9.47 & 0.90 & 3.34 & 0.87 \\
\hline
\end{tabular}

Table.8 Effect of integrated nitrogen management on available micronutrients

\begin{tabular}{|c|c|c|c|c|c|}
\hline \multirow[t]{2}{*}{ Symbol } & \multirow[t]{2}{*}{ Treatments } & \multicolumn{4}{|c|}{ Available micronutrients $\left(\mathrm{mg} \mathrm{kg}^{-1}\right)$} \\
\hline & & $\mathbf{Z n}$ & $\mathbf{F e}$ & $\mathbf{C u}$ & Mn \\
\hline $\mathbf{T}_{1}$ & Control & 0.99 & 2.29 & 0.46 & 7.47 \\
\hline $\mathbf{T}_{2}$ & $100 \% \mathrm{RDF}$ & 1.21 & 2.43 & 0.52 & 8.53 \\
\hline $\mathbf{T}_{\mathbf{3}}$ & $75 \% \mathrm{RDNF}+25 \% \mathrm{~N}(\mathrm{VC})$ & 2.63 & 3.81 & 0.63 & 10.86 \\
\hline $\mathbf{T}_{4}$ & $50 \% \mathrm{RDNF}+50 \% \mathrm{~N}(\mathrm{VC})$ & 2.95 & 4.28 & 0.72 & 12.44 \\
\hline $\mathbf{T}_{5}$ & $75 \%$ RDNF + 25\% N (FYM) & 2.44 & 3.53 & 0.58 & 10.14 \\
\hline $\mathbf{T}_{6}$ & $50 \% \mathrm{RDNF}+50 \% \mathrm{~N}(\mathrm{FYM})$ & 2.76 & 3.98 & 0.67 & 11.68 \\
\hline $\mathbf{T}_{7}$ & $50 \% \mathrm{RDNF}+25 \% \mathrm{~N}(\mathrm{VC})+25 \% \mathrm{~N}(\mathrm{FYM})$ & 2.86 & 4.14 & 0.69 & 11.95 \\
\hline \multirow[t]{2}{*}{$\mathbf{T}_{8}$} & $\begin{array}{l}75 \% \mathrm{RDNF}+12.5 \% \mathrm{~N}(\mathrm{VC})+12.5 \% \mathrm{~N} \\
(\mathrm{FYM})\end{array}$ & 2.54 & 3.65 & 0.60 & 10.55 \\
\hline & $\mathrm{CD}(\mathrm{p}=0.05)$ & 0.21 & 0.31 & 0.05 & 0.85 \\
\hline
\end{tabular}


Table.9 Effect of integrated nitrogen management on economic analysis

\begin{tabular}{|c|c|c|c|c|c|}
\hline Symbol & Treatments & $\begin{array}{c}\text { Total input } \\
\text { cost } \\
\left(\text { Rs. ha }{ }^{-1}\right)\end{array}$ & $\begin{array}{c}\text { Gross } \\
\text { returns } \\
\left(\text { Rs. } \text { ha }^{-1}\right)\end{array}$ & $\begin{array}{l}\text { Net returns } \\
\left(\text { Rs. ha }{ }^{-1}\right)\end{array}$ & B:C ratio \\
\hline $\mathbf{T}_{1}$ & Control & 110732 & 202577 & 91845 & 0.82 \\
\hline $\mathbf{T}_{2}$ & $100 \%$ RDF & 117918 & 378857 & 260939 & 2.21 \\
\hline $\mathbf{T}_{3}$ & $\begin{array}{l}75 \% \text { RDNF }+25 \% \\
N(\text { VC) }\end{array}$ & 124685 & 357283 & 232598 & 1.87 \\
\hline $\mathbf{T}_{4}$ & $\begin{array}{l}50 \% \text { RDNF + } 50 \% \\
N(\text { VC) }\end{array}$ & 132236 & 319177 & 186941 & 1.41 \\
\hline $\mathbf{T}_{5}$ & $\begin{array}{l}75 \% \text { RDNF + 25\% } \\
\text { N (FYM) }\end{array}$ & 120108 & 346737 & 226629 & 1.89 \\
\hline $\mathbf{T}_{6}$ & $\begin{array}{l}50 \% \text { RDNF + 50\% } \\
\text { N (FYM) }\end{array}$ & 121537 & 311777 & 190240 & 1.57 \\
\hline $\mathbf{T}_{7}$ & $\begin{array}{l}50 \% \text { RDNF }+25 \% \\
\text { N (VC) }+25 \% \mathrm{~N} \\
(\mathrm{FYM})\end{array}$ & 127735 & 315067 & 187332 & 1.47 \\
\hline $\mathbf{T}_{8}$ & $\begin{array}{l}75 \% \text { RDNF }+12.5 \% \\
\mathrm{~N}(\mathrm{VC})+12.5 \% \mathrm{~N} \\
(\mathrm{FYM})\end{array}$ & 120955 & 349243 & 228288 & 1.89 \\
\hline
\end{tabular}

In integrated nutrient management treatment available sulphur was recorded higher because organic manures enhance the activity of soil microorganisms and they apparently utilize organically bound sulphur.

They further convert it into sulphur containing amino acids like cystine and methionine, which are then converted in to inorganic sulphate by the action of microorganisms and increase the availability of sulphur in soil (Blum et al., 2013). Similarly, exchangeable calcium and magnesium was found significantly higher $58.47 \mathrm{mg} \mathrm{kg}^{-1}$ and $11.87 \mathrm{mg} \mathrm{kg}^{-1}$, respectively in $50 \% \mathrm{RDNF}+50 \% \mathrm{~N}$ (VC) applied treatment (Table 7). However, vermicompost was well known for improving soil properties by release of $\mathrm{Ca}$ and $\mathrm{Mg}$ from exchangeable sites (Uz et al., 2016).

Application of $50 \%$ RDNF $+50 \% \mathrm{~N}$ (VC) also documented with significant higher availability of cationic micronutrients ( $\mathrm{Zn}, \mathrm{Fe}$,
$\mathrm{Cu}$ and $\mathrm{Mn})$ and at par with treatments $\left(\mathrm{T}_{7}\right.$ and $\mathrm{T}_{6}$ ) in which $50 \% \mathrm{~N}$ was substituted through FYM and VC (Table 8). The increase in availability of cationic micronutrients may be attributed to decomposition of organic sources, which increased the availability of micronutrients by preventing fixation, oxidation, precipitation and leaching (Rai et al., 2018).

\section{Economic analysis}

The highest benefit: cost ratio of 2.21 was recorded in treatment $\mathrm{T}_{2}$ in which $100 \%$ recommended dose of nutrients was applied through fertilizers (Table 9). While, minimum benefit: cost ratio of 0.82 was observed in $T_{1}$ (control). In $T_{2}$ benefit: cost ratio was recorded highest due to reason that maximum yield was obtained with least investment as compared to other treatments in which only inorganic fertilizers were used in combination with organic manures. 
It is concluded that with the use of organic inputs through integrated nutrient management in the present study had marked effect in improving soil health and economic yield of cauliflower. Available macronutrients and cationic micronutrients $(\mathrm{Zn}, \mathrm{Fe}, \mathrm{Cu}$ and $\mathrm{Mn})$ were observed highest in plots where $50 \% \mathrm{~N}$ was substituted through FYM and VC. However, crop yield was highest in chemically fertilized plots $\left(\mathrm{T}_{2}\right)$. Application of $75 \% \mathrm{RDNF}+25 \% \mathrm{~N}$ through $\mathrm{VC}\left(\mathrm{T}_{3}\right)$ resulted in improved soil properties over entirely chemically fertilized plots without significant decrease in yield. Therefore, this treatment is recommended as beneficial for farmers on long term basis, as it resulted in saving $25 \%$ inorganic fertilizers for better net returns.

\section{References}

Anonymous (2017). Indian Fertilizer Scenario-2017. Department of Fertilizers, Ministry of Chemicals and Fertilizers, Government of India.

Blum, S.C., Lehmann, J., Solomon, D., Caires, E.F. and Alleoni, L.R.F. 2013. Sulphur forms in organic substrates affecting $\mathrm{S}$ mineralization in soil. Geoderma, 200-201: 156-164.

Bozkurt, S., Uygur, V., Agca, N. and Yalcin, M. 2011. Yield responses of cauliflower (Brassica oleracea L. var. botrytis) to different water and nitrogen levels in a Mediterranean coastal area. Soil and Plant Science, 61: 183-194.

Chander, G., Verma, T.S. and Sharma, S. 2010. Nutrient content of cauliflower (Brassica oleracea var. Botrytis L.) as influenced by boron and farm yard manure in north-west Himalayan alfisols. Journal of the Indian Society of Soil Science, 58: 248-251.

Chapman, H.D. and Pratt, P.F. 1961. Methods of analysis for soils, plants and waters, University of California, Divison of Agricultural Sciences, U.S.A.

Chaudhary, M.M., Bhanvadia, A.S. and Parmar, P.N. (2015). Effect of integrated nutrient management on growth, yield attributes and yield of cabbage (Brassica oleracea var. capitata L.) under middle Gujrat conditions. Trends in Biosciences, 8: 2164-2168.

Chesnin, L. and Yien, C.H. 1950. Turbidimetric determination of available sulphates. Soil Science Society of America Proceedings, 15: 149-151.

Devi, M., Upadhyay, G.P., Garima and Sephia, R.S. (2017). Biological properties of soil and nutrient uptake in cauliflower (Brassica oleracea var. botrytis L.) as influenced by integrated nutrient management. Journal of Pharmacognosy and Phytochemistry, 6(3): 325-328.

Dilz, K. 1988. Efficiency of uptake and utilization of fertilizer nitrogen by plant. In: Jenkinson, D.S. and Smith, K.A. (ed). Nitrogen efficiency in agricultural soils. pp. 1-26. Elsevier Applied Science, London.

Fageria, N.K., Dos santos, A.B. and Moraes, M.F. 2010. Influence of urea and ammonium sulphate on soil acidity indices in lowland rice production. Communication in Soil Science and Plant Analysis, 4: 1565-1575.

Jackson, M.L. (1987). Soil Chemical Analysis, Prentice-Hall of India, Private Limited, New Delhi.

Joshi, R., Singh, J. and Vig, A.P. 2015. Vermicompost as an effective organic fertilizer and biocontrol agent: effect on growth, yield and quality of plants. Reviews in Environmental Science and Biotechnology, 14: 137-159.

Kebrom, T.H. 2017. A growing stem inhibits 
bud outgrowth- The overlooked theory of apical dominance. Frontiers in Plant Science, 8: 1-7.

Kumar, A., Tripathi, H.P. and Yadav, D.S. 2007. Correcting nutrient for sustainable crop production. Indian Journal of Fertilizers, 2: 37-44.

Kumar, B. and Mukhopadhyay, S.K. 2017. Effect of integrated nutrient management on system productivity, nutrient uptake, nitrogen balance, soil structural properties and nitrogen use efficiency under wheat-rice cropping system. Journal of Pharmacognosy and Phytochemistry, SP1: 1030-1033.

Kumari, S. 2017. Effects of nitrogen levels on anatomy, growth and chlorophyll content in sunflower (Helianthus aпnииs L.) leaves. Journal of Agricultural Science, 9: 208-219.

Lindsay, W.L. and W.A. Norvell. 1978. Development of DTPA Soil test method for zinc, iron, manganese and copper. Soil Science Society of America Journal, 42: 421-428.

Merentola, Kanaujia, S.P. and Singh, V.B. 2012. Effect of integrated nutrient management on growth, yield and quality of cabbage (Brassica oleracea var. capitata). Journal of Soils and Crops, 22: 233-239.

Merwin, H.D. and Peech, M. 1951. Exchangeability of soils potassium in the sand, silt and clay fractions as influenced by the nature of complementary exchangeable cations. Soil Science Society of America Proceedings, 15: 125-128.

Novoa, R. and Loomis, R.S. 1981. Nitrogen and plant production. Plant Soil, 58: 177-204.

Ogbede, S.C., Saidu, A.N., Kabiru, A.Y. and Busari, M.B. 2015. Nutrient and antinutrient compositions of Brassica oleracea var. capitata L. International Organization of Scientific Research
Journal of Pharmacy, 5: 19-25.

Ohyama, T. 2010. Nitrogen as a major essential element of plants. Research Signpost, 37/661: 695-723.

Olsen, S.R., Cole, C.V., Watanabe, F.S. and Dean, L.A. 1954. Estimation of available phosphorus by extraction with sodium bicarbonate, U.S.D.A Circular, 939: 1-19.

Rai, A.P., Tundup, P., Mondal, A.K., Kumar, V., Samanta, A., Kumar, M., Arora, R.K. and Dwivedi, M.C. 2018. Cationic micronutrient status of some soils under different cropping system of Kishtwar district ( $\mathrm{J} \& \mathrm{~K}$ ), India. International Journal of Current Microbiology and Applied Sciences, 7: 3596-3602.

Rather, A.M., Jabeen, N., Bhat, T.A., Parray, E.A., Hajam, M.A. and Bhat, I.A. 2018. Effect of organic manures and biofertilizers on growth and yield of lettuce. The Pharma Innovation, 7: 7577.

Reza, Md. S., Islam, A.K.M.S., Rahman, Md.A., Miah, Md., Akhter, S. and Rahman, Md.M. 2016. Impact of organic fertilizers on yield and nutrient uptake of cabbage (Brassica oleracea var. capitata). Journal of Science, Technology and Environment informatics, 3: 231-244.

Scotti, R., Bonanomi, G., Scelza, R., Zoina, A. and Rao, M.A. 2015. Organic amendments as sustainable tool to recover fertility in intensive agriculture systems. Journal of Soil Science and Plant Nutrition, 15: 333352.

Singh, B. 2018. Are Nitrogen Fertilizers Deleterious to Soil Health? Agronomy, 8: 48.

Subbiah, B.V. and Asija, G.L. 1965. A rapid procedures for the estimation of available nitrogen in soils. Current Science, 25: 259-260. 
Tekasangla, Kanaujia, S.P. and Singh, P.K. 2015. Integrated nutrient management for quality production of cauliflower in acid alfisols of Nagaland. Karnataka Journal of Agricultural Sciences, 28: 244-247.

Uz, I., Sonmez, S., Tavali, I.E., Citak, S., Uras, D.S. and Citak, S. 2016. Effect of vermicompost on chemical and biological properties of an alkaline soil with high lime content during celery (Apium Graveolens L. var. deulce Mill.) production. Notulae Botanicae Horti Agrobotanici ClujNapoca, 44: 280-290.

Walkley, A. and Black, A. 1934. An examination of the Degtjareff method for determining soil organic matter and a proposed modification of the chromic acid titration method. Soil Sciences, 37: 27-38.

\section{How to cite this article:}

Harmandeep Singh Chahal, Satnam Singh, Iqbal Singh Dhillon and Simerpreet Kaur. 2019. Effect of Integrated Nitrogen Management on Macronutrient Availability under Cauliflower (Brassica oleracea var. botrytis L.). Int.J.Curr.Microbiol.App.Sci. 8(04): 1623-1633. doi: https://doi.org/10.20546/ijcmas.2019.804.189 